\title{
Enfermedad de Chagas y su seroprevalencia en tres departamentos de la Amazonia colombiana.
}

\author{
Chagas disease and its seroprevalence in three departments \\ of the Colombian Amazon \\ Carolina Flórez ${ }^{1}$, Lesly Guasmayan ${ }^{1}$, Liliana Cortés ${ }^{1}$, Andrés Caicedo ${ }^{1}$, Mauricio Beltrán ${ }^{2}$, Lyda Muñoz ${ }^{1}$.
}

\section{Resumen}

Objetivo. Determinar la seroprevalencia de la enfermedad de Chagas en población general procedente de tres departamentos de la Amazonía colombiana: Vaupés, Amazonas y Guaviare y analizar variables de riesgo para la enfermedad. Métodos. Para determinar la seropositividad se analizaron 3429 muestras de suero obtenidas mediante previo consentimiento informado durante los años 2009 y 2010 a través de un muestreo probabilístico, de conglomerados, estratificado y trietápico para cada departamento, con probabilidades finales desiguales. Fueron analizadas en el Laboratorio de Parasitología del Instituto Nacional de Salud de Bogotá mediante dos técnicas de diagnóstico, Inmunoensayo enzimático (Elisa) e Inmunofluorescencia indirecta (IFI) empleando como antígeno una cepa de Trypanosoma cruzi colombiana previamente caracterizada como linaje $T$ cI. Resultados. Se encontró una seroprevalencia general de 0,99\%, 2,07\% para el departamento del Guaviare, 0,79\% para el departamento de Vaupés y 0,09\% para el departamento de Amazonas. Estos resultados permitirán establecer una línea de base epidemiológica que contribuya a las estrategias de control de la enfermedad en esta zona.

Palabras clave: Seroprevalencia, Chagas, Amazonia, Trypanosoma cruzi

\section{Abstract}

Objective. To estimate the prevalence of Chagas disease in population from Vaupés, Amazonas and Guaviare, three departments of the Colombian amazon. Risk factors were also assessed. Methods. For estimating seroprevalence, 3429 serum samples were taken according to a threestage conglomerate sampling for each department. Those samples were analyzed in the Parasitology Laboratory of the National Health Institute (INS), through ELISA and IFAT techniques. Results. The prevalence for Amazonas, Guaviare and Vaupés departments was 0,09\%, 2,07\% and 0,79\%, respectively. Those results will allow health policy makers towards prevention of Chagas disease.

Keywords: Chagas disease, seroprevalence, Trypanosoma cruzi, Amazon

\footnotetext{
1. Grupo de Parasitología. Laboratorio Nacional de Referencia. Instituto Nacional de Salud. Bogotá. Colombia

2. Dirección Redes en Salud Pública. Instituto Nacional de Salud. Bogotá. Colombia

Correspondencia: aflorez@ins.gov.co

Fecha de recepción: Junio 27 de 2016 - Fecha de aprobación: Octubre 18 de 2016
} 


\section{Introducción}

La enfermedad de Chagas en la Amazonia es considerada una infección enzoótica entre animales y vectores silvestres y una antropozoonosis por invasión del hombre al ecótopo silvestre y/o cuando su domicilio es invadido por vectores y animales silvestres (1).

Algunas investigaciones serológicas realizadas en la Amazonía colombiana han demostrado seropositividades de $4,7 \%$ y en poblaciones indígenas hasta del 27\%. Debido al hallazgo de casos agudos en esta zona con alta intervención humana que produce deforestación. (2)

La relación ecológica de palmeras que contienen colonias de vectores y la dinámica de transmisión a la población humana por vectores silvestres que invaden casas sin llegar a colonizarlas es un fenómeno bien establecido (2).

La Amazonia contiene de manera natural gran variedad de vectores y reservorios infectados con T. cruzi, que generan ciclos de transmisión en focos naturales selváticos; invasión de domicilios por vectores silvestres altamente infectados (60\%), adaptación gradual de vectores como Triatoma maculata, ambiente domiciliario y patrones de baja transmisión hipoendémica de $T$. cruzi con seroprevalencias que varían entre 1 y $3 \%$. Se han documentado más de 500 casos graves de cardiopatía chagásica autóctonos en la Amazonia y a lo largo de los años se han incrementado los registros patológicos y la dimensión del impacto en la salud (3).

En países como Brasil se ha recogido toda la casuística sobre infectados en fase aguda en la región amazónica y se han descrito estudios de seroprevalencia en población general que van desde $2,8 \%$ hasta $5 \%$ y de 0,9 a $1,2 \%$ en algunos estados de la Amazonia brasilera (1, 4).

Algunas investigaciones serológicas aisladas realizadas en la zona de la Amazonia de Colombia han demostrado seropositividades de $4,7 \%$ y en pobla- ciones indígenas hasta del 27\% (5). El diagnóstico de casos agudos se ha incrementado en la Amazonia, especialmente en la zona de alta intervención humana que produce deforestación (6).

La zona Amazónica en Colombia corresponde a un área en la cual la enfermedad de Chagas ha sido poco explorada. A partir del año 2004 se han implementado algunas estrategias como la Iniciativa de los países amazónicos para la vigilancia y el control de la Enfermedad de Chagas (AMCHA) a través de la cual se han generado políticas de control, prevención, manejo e investigación de la enfermedad (7).

En prevención se destaca el tamizaje del 100\% de unidades de sangre en el departamento de Caquetá. En un trabajo realizado por Hilarión y colaboradores se realizó la caracterización epidemiológica de los casos reactivos (sospechosos) y confirmados para $T$. cruzi en donantes de sangre de dicho departamento desde 1995 a 2010, encontrándose que de 42.516 donaciones tamizadas, se detectaron 260 reactivas $(0,6 \%)$, de estas se confirmaron $116(45 \%)$ y 95 (82\%) resultaron positivos para T. cruzi (8).

En Colombia se han presentado brotes de transmisión oral desde el año 2008 notificados al Sistema de Vigilancia (SIVIGILA) del Instituto Nacional de Salud (9) y se han desarrollado algunos estudios de epidemiologia molecular relacionados con estos brotes que han demostrado una alta prevalencia de los genotipos de $T$. cruzi $T_{\mathrm{cI}}$ y $T_{\mathrm{cIV}}$, los cuales han sido aislados en otros brotes de transmisión oral, están relacionados con ciclos de transmisión selvática y juegan un papel muy importante en la interacción de hospederos selváticos y domésticos en el mismo nicho ecológico (10-12). Ninguno de estos brotes se ha presentado en departamentos de la Amazonia. Sin embargo, esto no implica que la patología esté ausente en esta región ya que se han obtenido datos de pacientes aislados con cuadros agudos procedentes de departamentos que conforman esta zona, los cuales igualmente han sido notificados al SIVIGILA (9). 
La transmisión vectorial representa un problema de salud pública y la selva de la Amazonia colombiana presenta un clima frecuentemente húmedo y caluroso durante todo el año que registra muy pocos triatominos domiciliados. Sin embargo, estudios de distribución geográfica y epidemiología han demostrado que Rhodnius brethesi, $R$. prolixus y $R$. pictipes son las especies silvestres en esta zona de mayor distribución (13).

La seroprevalencia en población general en la zona de la Amazonia colombiana se desconoce y con este estudio se contribuye al conocimiento de la seroprevalencia de la enfermedad de Chagas en población general procedente de tres departamentos de la Amazonia, lo que permitirá establecer una línea de base epidemiológica que apoyará las diferentes estrategias de control.

\section{Materiales y Métodos}

Fueron seleccionadas 3429 muestras de suero, a partir de un estudio de seroprevalencia poblacional de corte transversal, procedentes de personas entre los 2 a 64 ańos de edad de los departamentos de Vaupés, Amazonas y Leticia de la Amazonia colombiana.

Se utilizó un método de muestreo aleatorio, estratificado o por conglomerados y polietápico, en el que las muestras, previo consentimiento informado, fueron obtenidas durante los años 2009 y 2010, a través de un muestreo probabilístico, en el cual cada hogar censado tenía la probabilidad de selección superior a cero.

Los conglomerados correspondientes a los municipios, llamados Unidades Primarias de Muestreo (UPM) fueron seleccionados a partir de los municipios con más de 5000 habitantes que dispusieran de centro de salud y estratificados de acuerdo al nivel de urbanización, el índice de Necesidades Básicas Insatisfechas (NBI) y la composición urbana y rural de la población.
En un segundo tipo de conglomerados fueron establecidas áreas urbanas (manzanas) y áreas rurales (secciones censales) con un mínimo de diez domicilios residenciales (viviendas) y fueron llamadas Unidades Secundarias de Muestreo (USM), seleccionadas mediante la construcción de una frecuencia acumulada de USM separadas por zona y un cálculo de intervalos de muestreo en función del total de viviendas urbanas y rurales de los estratos de los cuales provenían, las que fueron ubicadas a través de la cartografía disponible en el Departamento Administrativo Nacional de Estadística (DANE) y un levantamiento complementario para la partición de los sectores censales, elegidos para la fácil localización y recorrido de los segmentos por parte de los encuestadores (14).

Se realizó finalmente un submuestreo en el terreno de las unidades USM con el levantamiento del listado de viviendas existentes en cada una de ellas, seleccionando aleatoria y sistemáticamente diez viviendas y sólo una persona del hogar que cumpliera con los requisitos establecidos en el estudio, previo alistamiento de todas las personas presentes en el momento de la visita.

Realizada la entrevista a la persona seleccionada o su acudiente responsable, mediante aplicación de un instrumento de recolección de datos diseñado para tal fin y extraída una muestra de sangre venosa de $5 \mathrm{ml}$, con el diligenciamiento previo de un consentimiento informado, la información fue recogida e incluida en una base de datos para realizar posteriormente su análisis, en el cual cada valor muestral de cada encuesta fue ponderado por el recíproco de la probabilidad final de cada persona, que es el denominado factor de expansión de la muestra al universo. Dicho factor, por un lado, corrige el sesgo que generan las probabilidades desiguales, y por otro permite la estimación de datos absolutos para el universo estudiado. La probabilidad final, calculada con base en la muestra encuestada, supone que la muestra seleccionada no encuestada, forma parte del universo y que sus características son similares, en promedio, a las de la muestra encuestada. 
Las muestras de sangre fueron recolectadas mediante venopunción (investigación considerada como de Riesgo Mínimo de acuerdo a la Resolución No 008430 del Ministerio de Salud) y fueron almacenadas en condiciones de congelación a $-70^{\circ} \mathrm{C} \pm 5^{\circ} \mathrm{C}$ hasta su procesamiento.

Para determinar la seropositividad fueron analizadas en el Laboratorio de Parasitología del Instituto Nacional de Salud (INS) de Bogotá la técnica convencional de Inmunoensayo enzimático (Elisa) según metodología del INS (15) y como prueba complementaria la técnica convencional de Inmunofluorescencia indirecta (IFI) empleando extractos antigénicos de cepas colombianas de Trypanosoma cruzi previamente caracterizadas como linaje $T_{\mathrm{CI}}$ (16).

\section{Resultados}

Fueron procesadas 3429 muestras de suero de personas procedentes de tres departamentos de la Amazonia colombiana, Vaupés (1137), Guaviare (1162) y Amazonas (1130). Se describen las características de la población de estudio en la Tabla 1 .

Tabla 1. Características de la población de estudio de tres departamentos de la Amazonia colombiana

\begin{tabular}{|c|c|c|c|c|c|c|c|c|c|}
\hline \multirow[t]{2}{*}{ VARIABLE } & \multirow[t]{2}{*}{ SUB-VARIABLE } & \multicolumn{2}{|c|}{$\begin{array}{l}\text { VAUPÉS } \\
\mathbf{n}=1137\end{array}$} & \multicolumn{2}{|c|}{$\begin{array}{c}\text { GUAVIARE } \\
\mathrm{n}=1162\end{array}$} & \multicolumn{2}{|c|}{$\begin{array}{c}\text { AMAZONAS } \\
\mathrm{n}=1130\end{array}$} & \multicolumn{2}{|c|}{$\begin{array}{l}\text { TOTAL } \\
\mathbf{n}=3429\end{array}$} \\
\hline & & \multicolumn{2}{|c|}{$\mathbf{n}$} & \multicolumn{2}{|c|}{$\mathbf{n}$} & \multicolumn{2}{|r|}{$\mathbf{n}$} & \multicolumn{2}{|c|}{$\mathbf{n}$} \\
\hline \multirow{2}{*}{ SEXO } & Femenino & 623 & $54,6 \%$ & 779 & $67,0 \%$ & 742 & $65,7 \%$ & 2144 & $62,5 \%$ \\
\hline & Masculino & 514 & $45,1 \%$ & 383 & $33,0 \%$ & 388 & $34,3 \%$ & 1285 & $37,5 \%$ \\
\hline \multirow{4}{*}{ EDAD } & Edad 2-18 & 180 & $15,8 \%$ & 160 & $13,8 \%$ & 127 & $11,2 \%$ & 467 & $13,6 \%$ \\
\hline & Edad 19-30 & 362 & $31,8 \%$ & 350 & $30,1 \%$ & 394 & $34,9 \%$ & 1106 & $32,3 \%$ \\
\hline & Edad $31-50$ & 419 & $36,8 \%$ & 462 & $39,8 \%$ & 430 & $38,1 \%$ & 1311 & $38,2 \%$ \\
\hline & Edad 51-64 & 176 & $15,4 \%$ & 190 & $16,4 \%$ & 179 & $15,8 \%$ & 545 & $15,9 \%$ \\
\hline \multirow{4}{*}{$\begin{array}{c}\text { ESTRATO } \\
\text { SOCIOECONÓMICO }\end{array}$} & Estrato 0 y 1 & 950 & $83,3 \%$ & 617 & $53,1 \%$ & 524 & $46,4 \%$ & 2091 & $61,0 \%$ \\
\hline & Estrato 2 y 3 & 186 & $16,3 \%$ & 544 & $46,8 \%$ & 595 & $52,7 \%$ & 1325 & $38,6 \%$ \\
\hline & Estrato 4 & 1 & $0,1 \%$ & 0 & $0,0 \%$ & 11 & $1,0 \%$ & 12 & $0,3 \%$ \\
\hline & Sin dato & 0 & 0 & 1 & $0,1 \%$ & 0 & $0,0 \%$ & 1 & $0,0 \%$ \\
\hline \multirow{5}{*}{ ESCOLARIDAD } & Sin escolaridad & 70 & $6,1 \%$ & 74 & $6,4 \%$ & 47 & $4,2 \%$ & 191 & $5,6 \%$ \\
\hline & $\begin{array}{c}\text { Preescolar o hasta } \\
\text { Primaria }\end{array}$ & 359 & $31,5 \%$ & 476 & $41,0 \%$ & 355 & $31,4 \%$ & 1190 & $34,7 \%$ \\
\hline & $\begin{array}{c}\text { Secundaria incompleta o } \\
\text { culminada }\end{array}$ & 550 & $48,2 \%$ & 489 & $42,1 \%$ & 613 & $54,2 \%$ & 1652 & $48,2 \%$ \\
\hline & $\begin{array}{c}\text { Algún grado de } \\
\text { educación superior }\end{array}$ & 158 & $13,9 \%$ & 121 & $10,4 \%$ & 111 & $9,8 \%$ & 390 & $11,4 \%$ \\
\hline & Sin dato & 0 & 0 & 2 & $0,2 \%$ & 4 & $0,4 \%$ & 6 & $0,2 \%$ \\
\hline \multirow{3}{*}{$\begin{array}{l}\text { UBICACIÓN } \\
\text { VIVIENDA }\end{array}$} & Vivienda urbana & 877 & $76,9 \%$ & 974 & $83,8 \%$ & 770 & $68,1 \%$ & 2621 & $76,4 \%$ \\
\hline & Vivienda rural & 140 & $12,3 \%$ & 109 & $9,4 \%$ & 360 & $31,9 \%$ & 609 & $17,8 \%$ \\
\hline & Cabecera municipal & 120 & $10,5 \%$ & 79 & $6,8 \%$ & 0 & $0,0 \%$ & 199 & $5,8 \%$ \\
\hline \multirow{4}{*}{$\begin{array}{l}\text { PISO DE LA } \\
\text { VIVIENDA }\end{array}$} & Piso Baldosa o cemento & 475 & $41,7 \%$ & 955 & $82,2 \%$ & 646 & $57,2 \%$ & 2076 & $60,5 \%$ \\
\hline & Piso Tierra & 280 & $24,6 \%$ & 138 & $11,9 \%$ & 16 & $1,4 \%$ & 434 & $12,7 \%$ \\
\hline & Piso madera & 349 & $30,6 \%$ & 46 & $4,0 \%$ & 464 & $41,1 \%$ & 859 & $25,1 \%$ \\
\hline & Otro (madera-barro) & 33 & $2,9 \%$ & 23 & $2,0 \%$ & 4 & $0,4 \%$ & 60 & $1,7 \%$ \\
\hline \multirow{2}{*}{$\begin{array}{l}\text { PAREDES DE LA } \\
\text { VIVIENDA }\end{array}$} & Bahareque & 18 & $1,6 \%$ & 3 & $0,3 \%$ & 2 & $0,2 \%$ & 23 & $0,7 \%$ \\
\hline & Tapia Pisada & 1 & $0,1 \%$ & 3 & $0,3 \%$ & 2 & $0,2 \%$ & 6 & $0,2 \%$ \\
\hline
\end{tabular}




\begin{tabular}{|c|c|c|c|c|c|c|c|c|c|}
\hline \multirow[t]{2}{*}{ VARIABLE } & \multirow[t]{2}{*}{ SUB-VARIABLE } & \multicolumn{2}{|c|}{$\begin{array}{l}\text { VAUPÉS } \\
\mathrm{n}=1137\end{array}$} & \multicolumn{2}{|c|}{$\begin{array}{c}\text { GUAVIARE } \\
\mathrm{n}=1162\end{array}$} & \multicolumn{2}{|c|}{$\begin{array}{c}\text { AMAZONAS } \\
\mathrm{n}=1130\end{array}$} & \multicolumn{2}{|c|}{$\begin{array}{l}\text { TOTAL } \\
\mathrm{n}=3429\end{array}$} \\
\hline & & \multicolumn{2}{|c|}{$\mathrm{n}$} & \multicolumn{2}{|r|}{ n } & \multicolumn{2}{|r|}{ n } & \multicolumn{2}{|c|}{$\mathrm{n}$} \\
\hline \multirow{2}{*}{$\begin{array}{c}\text { PAREDES DE LA } \\
\text { VIVIENDA }\end{array}$} & Bloque o Ladrillo & 228 & $20,0 \%$ & 867 & $74,6 \%$ & 562 & $49,7 \%$ & 1657 & $48,3 \%$ \\
\hline & Otro (madera) & 890 & $78,1 \%$ & 289 & $24,9 \%$ & 564 & $49,9 \%$ & 1743 & $50,8 \%$ \\
\hline \multirow{5}{*}{$\begin{array}{l}\text { TECHO DE LA } \\
\text { VIVIENDA }\end{array}$} & Zinc & 1053 & $92,4 \%$ & 856 & $73,7 \%$ & 915 & $81,0 \%$ & 2824 & $82,4 \%$ \\
\hline & Eternit o Concreto & 34 & $3,0 \%$ & 219 & $18,8 \%$ & 96 & $8,5 \%$ & 349 & $10,2 \%$ \\
\hline & Cielo Raso & 16 & $1,4 \%$ & 33 & $2,8 \%$ & 103 & $9,1 \%$ & 152 & $4,4 \%$ \\
\hline & $\begin{array}{l}\text { Otro (plástico, paja, } \\
\text { madera) }\end{array}$ & 34 & $3,0 \%$ & 53 & $4,6 \%$ & 16 & $1,4 \%$ & 103 & $3,0 \%$ \\
\hline & Sin dato & 0 & 0 & 1 & $0,1 \%$ & 0 & $0,0 \%$ & 1 & $0,0 \%$ \\
\hline
\end{tabular}

La seroprevalencia general para la enfermedad de Chagas en los tres departamentos de la Amazonia colombiana fue de $0,99 \%$. Para el departamento del Guaviare fue 24 (2,07\%), para Vaupés 9 (0,79\%) y para el departamento de Amazonas 1 (0,09\%), siendo la más baja seroprevalencia hallada.

La distribución por sexo de la población seropositiva fue $55,8 \%$ pertenecientes al sexo femenino y $44,1 \%$ al masculino $(p>0.5)$. El 67,6\% de los seropositivos vivía en zona urbana de los municipios y el 32,4\% restante tenía su vivienda en zona rural y en la cabecera municipal.
En cuanto al lugar de nacimiento de los seropositivos, el $100 \%$ de los procedentes del departamento de Vaupés habían nacido allí, asimismo el 25\% de los procedentes de Guaviare habían nacido en municipios propios de este departamento y el 75\% restante eran naturales de departamentoscomoSantander, Boyacá, Cundinamarca, Caldas, Meta y Vaupés. El único seropositivo de procedencia del departamento de Amazonas era natural del departamento de Norte de Santander y el único seropositivo menor de edad correspondió a un infante de 7 ańos nacido y procedente del departamento de Vaupés. La mayor seroprevalencia $(2,57 \%)$ se presentó en el grupo de edad de 51 a 64 años. Tabla 2.

Tabla 2. Resultados de seroprevalencia para enfermedad de Chagas, por grupos de edad

\begin{tabular}{cccc|}
\hline Grupo etario (años) & $\begin{array}{c}\text { Total de } \\
\text { examinados }\end{array}$ & Positivos & \begin{tabular}{c} 
Seroprevalencia \\
\cline { 4 - 4 }
\end{tabular} \\
\hline Edad 2-18 & 467 & 1 & $(\%)$ \\
\hline Edad 18-30 & 1106 & 2 & 0,21 \\
\hline Edad 31 -50 & 1311 & 17 & 0,18 \\
\hline Edad 51-64 & 545 & 14 & 1,3 \\
\hline Total & 3429 & 34 & 0,57 \\
\hline
\end{tabular}

El 70.6\% (24) de los identificados como seropositivos pertenecían a estratos 0,1 . El 73,5\% (25) no tenía escolaridad o había cursado algún grado de básica primaria sin culminarla, el 20,6\% (7) había cursado algún grado de secundaria y solo el 5,9\% (2) tenía algún grado de educación superior. Asimismo, el 85,3\% (29) pertenecía al régimen subsidiado y en el 38,2\% (13) su vivienda tenía piso de tierra, barro o madera, en el $11,8 \%$ (4) las paredes eran de bahareque y madera y en el 5,9\% (2) el techo era de madera y paja.
Algunas variables fueron analizadas para determinar su posible asociación con la Enfermedad de Chagas, observando que ser mayor de 30 años y tener un grado de escolaridad muy bajo o no tener ningún grado resultaron ser factores de riesgo estadísticamente significativos. Otra variable, como pertenecer al régimen subsidiado en salud demostró tener una mayor asociación casi dos veces mayor, sin ser estadísticamente significativa. Tabla 3. 
Tabla 3. Variables asociadas con la presencia de Enfermedad de Chagas en tres departamentos de la Amazonia colombiana.

\begin{tabular}{|c|c|c|c|c|c|}
\hline Variable & Positivos & Negativos & $\mathbf{R R}$ & IC 95\% & $p$ \\
\hline Vivienda en zona rural y cabecera municipal & 11 & 797 & 1,55 & $0,76-3,17$ & \\
\hline Vivienda en zona urbana & 23 & 2598 & & & \\
\hline Ser mayor de 30 años & 31 & 1825 & 8,76 & $2,68-28,59$ & $<0,00 \ldots$ \\
\hline Ser menor de 30 años & 3 & 1570 & & & \\
\hline Sexo masculino & 15 & 1270 & 1,32 & $0,67-2,58$ & \\
\hline Sexo femenino & 19 & 2125 & & & \\
\hline Régimen Subsidiado en SGSS & 29 & 2253 & 2,92 & $1,13-7,51$ & \\
\hline Régimen Contributivo y especial & 5 & 1142 & & & \\
\hline Sin escolaridad o primaria incompleta & 25 & 1356 & 4,12 & $1,93-8,80$ & $<0,00 \ldots$ \\
\hline $\begin{array}{l}\text { Secundaria incompleta, completa o algún } \\
\text { grado de educación superior }\end{array}$ & 9 & 2039 & & & \\
\hline Piso de tierra, madera, barro & 13 & 2055 & 0,41 & $0,20-0,81$ & \\
\hline Piso de baldosa o cemento & 21 & 1340 & & & \\
\hline Paredes de bahareque, tapia pisada & 12 & 1760 & 0,51 & $0,25-1,03$ & \\
\hline Paredes de bloque, ladrillo, madera & 22 & 1635 & & & \\
\hline Techo de plástico, paja, madera & 2 & 102 & 2 & $0,49-8,23$ & \\
\hline Techo de zinc, eternit, concreto o cielo raso & 32 & 3293 & & & \\
\hline Estrato 0 y 1 & 24 & 2067 & 1,54 & $0,74-3,20$ & \\
\hline Estrato 2,3 y 4 & 10 & 1328 & & & \\
\hline
\end{tabular}

\section{Discusión}

La región amazónica de Colombia abarca casi el 40\% del territorio nacional. Se compone principalmente de bosques tropicales poco poblados y es considerada como de bajo riesgo para la Enfermedad de Chagas. Sin embargo, los datos sobre la epidemiología clínica de la enfermedad son escasos (3).

La seroprevelancia obtenida en este estudio fue menor a la hallada en otras regiones de la amazonia de países vecinos como Brasil, en el que se llevó a cabo un estudio de seroprevalencia en el cual se analizaron 4.880 muestras de sangre obtenidas en papel de filtro mediante la técnica de IFI encontrándose una tasa de positividad de 4,5\% para la infección de Chagas (17). Otro estudio en área amazónica occidental de Brasil reveló una tasa muy similar a la hallada en este estudio pare al departamento de Amazonas, con una seroprevalencia que osciló entre $0.9 \%$ a $1.2 \%$ (4).

Es importante resaltar que el incremento de casos autóctonos de la enfermedad de Chagas en la Amazonia a partir de los ańos setenta puede ser un indicadorquesugiereque, como en otraszonas del país, puede convertirse en un problema de salud pública en la región. Sin embargo, los cambios del patrón epidemiológico del evento en la región amazónica pueden ser explicados por las transformaciones ambientales y sociales que han sucedido, encauzados por los procesos de la globalización en términos de producción y comercio internacional. Aunque existe evidencia de notificación de casos, no existen registros de domiciliación de vectores en la dinámica de transmisión (18). 
La implementación de nuevas actividades como la agrícola, ganadera, minera y maderera, junto al desarrollo urbano, han generado cambiossignificativos en el medio ambiente y en el hábitat indígena tradicional y han provocado corrientes migratorias, deforestación, sedentarismo, domesticación de animales y modificaciones en el hábitat que facilitan la colonización de las viviendas por los vectores y por ende la transmisión doméstica y laboral de la enfermedad (19). Otros estudios también resaltan algunas variables relacionadas con la transmisión de la enfermedad, como la ventilación, iluminación, dormir junto a la pared, área de construcción anexos a la vivienda y la presencia de cobertizos $(20,21)$.

Además, la ocurrencia de la enfermedad de Chagas también puede estar sostenida en factores sociales como la pobreza, la migración, el tipo y calidad de vivienda y su ubicación en la localidad, así como la falta de participación comunitaria y los pocos conocimientos y prácticas sobre la misma enfermedad. Otros factores fundamentales como la falta de escolaridad y la dificultad en el acceso a los servicios de salud (los cuales demostraron una mayor asociación con la enfermedad en este estudio), pueden llegar a contribuir a la presencia de esta enfermedad en una región. Algunos factores conductuales como la carencia en el uso de medidas de protección personal y el descuido en horas de descanso propios de la cultura de cada comunidad, pueden igualmente aportar a su sostenimiento en los diferentes hábitats, así como los factores ecológicos como temperatura, humedad, pluviosidad y cobertura vegetal y sus posibles interacciones que propician condiciones necesarias para la sobrevivencia del vector y su componente entomológico (hábitos de picadura, sitios de reposo, tasas de paridad y criaderos) (22).

Por otra parte, en la epidemiología de la enfermedad de Chagas se considera que algunas características de las viviendas pueden beneficiar la infestación por vectores o por el contrario actuar como factores protectores y evitar su presencia. De este modo paredes con rendijas o superficies irregulares, techos de palma y pisos de tierra, los cuales sin ser estadísticamente significativos también fueron identificados en algunas viviendas de los seropositivos en este estudio, pueden proporcionar abrigos adecuados a estos insectos.

Otros elementos frecuentemente relacionados con la transmisión de la Enfermedad de Chagas son las carencias higiénicas, la desorganización en la vivienda, el hacinamiento, la convivencia con animales domésticos y silvestres y la falta de conocimiento de los factores de riesgo relacionada con la falta de escolaridad, entre otras (23-25). Sin embargo, otros autores afirman que los habitantes de la región amazónica tienen un amplio conocimiento del vector evidenciando que el 79\% de encuestados en un estudio realizado en zona amazónica, manifestaron conocerlo y el 100\% de estos reconocían el insecto con el nombre de pito. Asimismo, encontraron asociación positiva entre seropositividad anti T. cruzi con el hecho de reconocer el pito en pacientes que habían recibido marcapasos por cardiopatía chagásica (26).

Los resultados obtenidos en este estudio en cuanto a la distribución por edad, donde la mayoría de los casos sobrepasaban los 30 ańos, siendo estadísticamente significativo y la procedencia en el 56\% de los casos correspondió a zonas ubicadas fuera de los departamentos de Guaviare y Amazonas, sugiere que no existe una circulación persistente en estos departamentos de la Amazonia colombiana, considerando en general que a edades tempranas no se observa una seroprevalencia elevada y los casos fueron en su mayoría importados de zonas conocidas como endémicas para la enfermedad.

Los datos de infección de triatominos y pocas evidencias de domiciliación de los vectores de esta región probablemente pueden revelar que quizás los niños no sean la población objeto de vectores extradomiciliados y la infección con $T$. cruzi sea más potencial en individuos de mayor edad dedicados a actividades en donde realizan ingreso a los ecótopos naturales de los vectores (13). 
Esto se puede deber a que donde hay un control de la transmisión intradomiciliaria, el peridomicilio cobra importancia y se puede presentar una disminución de los casos agudos y tasas de prevalencia de la población joven, desplazando la morbi-mortalidad a franjas mayores de edad (27). Sin embargo, para el caso específico del departamento del Vaupés de la zona amazónica colombiana, sí se sugiere que existe probablemente la presencia de una infección autóctona, originada por la existencia de una verdadera zoonosis sostenida por los ciclos silvestres del lugar (3). El hecho de encontrar la infección de T. cruzi en un infante de 7 ańos y natural del departamento hace pensar en una posible transmisión activa en esta zona, la cual debe ser corroborada con nuevos estudios.

Las características de la Enfermedad de Chagas en la región amazónica requieren la adopción de un modelo de vigilancia diferente de lo que se ha seguido en las zonas históricamente endémicas de Colombia, el cual se centra fundamentalmente en la vigilancia entomológica. Del mismo modo, para consolidar un modelo de vigilancia y control de la enfermedad en esta región, es importante considerar la extensión del territorio del Amazonas y las dificultades operativas que esto implica; así como el hecho que la enfermedad no se reconoce como un problema de salud pública, por lo que es fundamental el mejoramiento de la gestión de recursos existentes en la región, con el objetivo de prevenir que la enfermedad de Chagas se convierta en endémica (28). Además es necesario activar el plan de detección de seropositivos menores de quince ańos, fortalecer la capacidad de manejo integral del paciente chagásico y continuar con la vigilancia de $T$. cruzi en vectores y reservorios.

El aporte de este estudio en Salud pública está principalmente orientado a la prevención donde la sensibilización hacia la enfermedad, el ordenamiento del medio y las medidas de protección personal juegan un papel importante ante la poca sostenibilidad de un programa de mejoramiento de vivienda. Los resultados deben tener un gran impacto en las campañas de sensibilización para la comunidad, unificando el lenguaje en las cartillas comunitarias o material para Información, educación y comunicación (IEC) en la zona, lo cual a su vez es la base para que las personas que se encuentran en riesgo de adquirir la infección tomen las medidas adecuadas de protección personal y de prevención para la infestación de las viviendas y así poder contribuir al control de los principales factores de riesgo asociados con el evento en esta zona.

Finalmente es importante precisar que, teniendo en cuenta el control integral del Programa de Enfermedad de Chagas en Colombia, el tratamiento etiológico fue administrado al infante diagnosticado en el departamento del Vaupés y los autores recomendaron el seguimiento de los 33 pacientes seropositivos restantes y disponer del tratamiento de acuerdo a criterio médico.

\section{Conflicto de intereses}

Los autores declaran que no existe conflicto de intereses.

Este trabajo fue financiado con recursos propios del Instituto Nacional de Salud. Bogotá, Colombia

\section{Agradecimientos}

Agradecemos el apoyo incondicional dado por parte de los funcionarios de las Secretarías de Salud de los municipios y departamentos, de los Hospitales públicos y de los Laboratorios Departamentales de Salud Pública. Y de manera especial agradecemos, en general, a toda la población que voluntariamente participó, sin la que no se hubiera podido realizar el estudio.

\section{Referencias}

1. Coura J, Junqueira A. Risks of endemicity, morbidity and perspectives regarding the control of Chagas disease in the Amazon Region Mem Inst Oswaldo Cruz. 2012; 107: 145-15.

2. Abad-Franch F, Ferraz G, Campos C, Palomeque FS, Grijalva MJ, Aguilar HM, et al. Modeling disease vector occurrence when detection 
is imperfect: infestation of Amazonian palm trees by triatomine bugs at three spatial scales. PLoSNegl Trop Dis. 2010;4: 620.

3. Aguilar HM, Abad-Franch F; Pinto Dias JC, Veríssimo AC Coura JC. Chagas Disease in Amazon Region. Mem. Inst. Oswaldo Cruz. 2007; 103:47-56. DOI: 10.1590/S007402762007005000098 .

4. Magalhães BM, Coelho LI, Maciel MG, Ferreira JM, Umezawa ES, Coura JR, et al. Serological survey for Chagas disease in the rural areas of Manaus, Coari and Teféin the Western Brazilian Amazon. Rev Soc Bras Med Trop. 2011; 44: 697-702.

5. Vélez I, Murcia L.M. Prospección epidemiológica de enfermedades tropicales en los indígenas Tikunas residentes en el río Pupuna -Amazonas. Documento Técnico Secretaría de Salud Departamental del Amazonas.1999.

6. Aguilar MD. Cambio climático local y emergencia de enfermedades vectoriales en la Amazonía. I Seminario Internacional de Cambio Climático y Salud. Una visión de la Mitad del Mundo. Memorias. Quito. 2013:124-132.

7. Organización Panamericana de la Salud (OPS). Iniciativa de los países amazónicos para la vigilancia y el control de la Enfermedad de Chagas (AMCHA). Washington D.C: OPS; 2004.

8. Hilarión L. Caracterización epidemiológica de todos los casos reactivos y confirmados de Trypanosoma cruzi en donantes de sangre del Departamento de Caquetá de 1995 a 2010. Tesis presentada como requisito parcial para optar al título de: Especialista en Administración en Salud Pública. Universidad Nacional de Colombia. 2013.

9. Sistema de Vigilancia (SIVIGILA) del Instituto Nacional de Salud. 2008-2013.

10. Marcili A, Lima L, Valente VC, Valente SA, Batista JS, Junqueira AC, et al. Comparative phylogeography of Trypanosoma cruzi TCIIc: New hosts, association with terrestrial ecotopes, and spatial clustering. Infect Genet Evol. 2009; 9:1265-74. doi: 10.1016/j.meegid.2009.07.003.

11. Zingales B, Miles MA, Campbell DA, Tibayrenc M, Macedo AM, Teixeira MM, et al. The revised Trypanosoma cruzi subspecific nomenclature: Rationale, epidemiological relevance and research applications. Infect Genet Evol. 2012; 12:240-53.

12. Ramírez JD, Montilla M, Cucunuba Z, Flórez A, Zambrano P, Guhl F. Molecular Epidemiology of Human Oral Chagas Disease Outbreaks in Colombia. PLoS Negl Trop Dis. 2013; 7: 2041.

13. Guhl F, Aguilera G, Pinto N, Vergara D. Actualización de la distribución geográfica y ecoepidemiología de la fauna de triatominos (Reduviidae: Triatominae) en Colombia. Biomédica. 2007; 27:143-62

14. Flórez A, Pastrán S, Vargas N, Beltrán M, Enriquez Y, Peńa A, et al. Cisticercosis en Colombia. Estudio de seroprevalencia 2008-2010. Acta Neurol Colomb. 2013; 29:73-86.

15. López M, Duque S, Orozco LC, Camargo D, Gualdrón L, Cáceres E, et al. Inmunodiagnóstico de la infección chagásica por ELISA. Biomédica. 1999; 19: 159 -63.
16. Camargo ME. Fluorescent antibodytest for the serodiagnosis of American trypanosomiasis. Thechnical modification employing preserved culture forms of Trypanosoma cruzi in a slide test. Rev Inst Med Trop Sao Paulo. 1966; 8:227-34

17. Coura J, Borges-Pereira J. Chagas disease. What is known and what should be improved: a systemic review. Rev Soc Bras Med Trop. 2012; 45:286-96.

18. Magalhães B, Câmara Coelho L, Maciel M, Ferreira JC, Umezawa E, Coura J, JA Vale Barbosa M. Serological survey for Chagas disease in the rural areas of Manaus, Coari, and Tefé in the Western Brazilian Amazon. Rev Soc Bras Med Trop. 2011; 44(6):697-702

19. Briceño-León R.Chagas disease and globalization of the Amazon. Cad Saúde Pública 2007; 23: s33-s40. doi. org/10.1590/S0102-311X2007001300005.

20. Campbell-Lendrum D, Angulo V, Esteban L,Tarazona Z, Parra G, Restrepo M, et al.

21. House-level risk factors for triatomine infestation in Colombia. Int J Epidemiol. 2007; 36: 866-72 doi:10.1093/ije/dym065.

22. Segura EL, Escobar-Mesa A. Epidemiología de la enfermedad de Chagas en el estado de Veracruz. Salud Pub Méx. 2005; 47: 201-8.

23. Govere J, Durrheim D, Grange KI, Mabuza A, Booman M Community knowledge and perceptions about malaria and practices influencing malaria control in Mpumalanga province, South Africa. S Afr Med J. 2000; 90: 611-6.

24. Sanmartino M, Crocco L. Conocimientos sobre la enfermedad de Chagas y factores de riesgo en comunidades epidemiológicamente diferentes de Argentina. Rev Panam Salud Pública; 2000; 7:173-7.

25. Crocco L, Rodríguez C, Catalá S, Nattero J. Enfermedad de Chagas en Argentina: herramientas para que los escolares vigilen y determinen la presencia de factores de riesgo en sus viviendas. Cad Saúde Pública; 2005; 21:646-51.

26. Manrique FG, Camacho SM, Saavedra DL, Herrera GM, Ospina JM. Prácticas de autocuidado en gestantes con riesgo de contraer enfermedad de Chagas en Moniquirá y Miraflores, Colombia. Rev Fac Nac Salud Pública; 2011. 28: 231-41.

27. Mora G, Echeverry MC, Rey GE, López MC, Posada LF, Rivas FA. Frecuencia de anticuerpos anti-Trypanosoma cruzi en pacientes portadores de marcapasos de la Clínica San Pedro Claver de Bogotá. Biomédica. 2007; 27:483-9

28. Castro M. Aspectos Ecoepidemiológicos en la transmisión de la Enfermedad de Chagas en Santa Rosalia, Vichada. Tesis presentada como requisito parcial para optar al título de: Magíster en Infecciones y Salud en el Trópico. Universidad Nacional de Colombia. 2013.

29. Silveira A. Group discussion: epidemiological and social determinants of Chagas disease and its control in the Amazon countries. Mem Inst Oswaldo Cruz. 2007; 102:71-74. 